\title{
Cestóides Trypanorhyncha parasitos de peroá, Balistes capriscus Gmelin, 1789 comercializados no estado do Rio de Janeiro, Brasil
}

\author{
Trypanorhyncha cestodes parasitic in grey-triggerfish, Balistes \\ capriscus Gmelin, 1789 purchased in Rio de Janeiro State, Brazil
}

\author{
Fátima de J. E. Dias, ${ }^{*}$ Sérgio C. de São Clemente, ${ }^{*}$ Marcelo Knoff**
}

\begin{abstract}
Resumo
Entre junho de 2005 e agosto de 2006 foram adquiridos 100 espécimes de peroá, Balistes capriscus Gmelin, 1789 , em estabelecimentos de pescado nos municípios de Niterói e Rio de Janeiro. Os peixes foram medidos, necropsiados, filetados e seus órgãos analisados. Destes espécimes analisados 9 peixes estavam parasitados por plerocercos pertencentes à espécie Callitetrarhynchus gracilis e C. speciosus, apresentando respectivamente, $9 \%$ e $2 \%$ de prevalência, 14,4 e 1 de intensidade média e 0,13 e 0,02 de abundância média, amplitude da variação da intensidade de infecção em $C$. gracilis foi de 1 a 32 e $C$. speciosus apresentou um parasito por peixe, sendo os sítios de infecção, respectivamente, mesentério e fígado, e mesentério. Estas duas espécies são relatadas pela primeira vez em $B$. capriscus.
\end{abstract}

Palavras-chave: Trypanorhyncha, Callitetrarhynchus gracilis, Callitetrarhynchus speciosus, Balistes capriscus.

\begin{abstract}
One hundred specimens of grey triggerfish, Balistes capriscus Gmelin, 1789 purchased from markets of municipalities of Niterói and Rio de Janeiro from June 2005 to August 2006. The fishes were measured, necropsied, fileted and analysed their orgarns. Nine fishes were parasitized by metacestodes of Trypanorhyncha, the collected species were Callitetrarhynchus gracilis and C. speciosus with respectivelly, $9 \%$ and $2 \%$ of prevalence, 14.4 and 1 of mean intensity, and 0.13 and 0.02 of mean abundance, the range of intensity of infection by $C$. gracilis were 1 to 32 and $C$. speciosus showed one parasite in each fish, and the sites of infection were respectivelly, mesentery and liver, and mesentery. These two species were reported in $B$. capriscus for the first time.
\end{abstract}

Keywords: Trypanorhyncha, Callitetrarhynchus gracilis, Callitetrarhynchus speciosus, Balistes capriscus.

\section{Introdução}

A espécie Balistes capriscus Gmelin, 1789, conhecido como peroá, peixe-porco, porquinho ou cangulo, ocorre em todo o litoral brasileiro e tem carne considerada de boa qualidade, além de custo mais acessível no mercado varejista, havendo poucas pesquisas referentes à sua fauna parasitológica e importância na higiene e inspeção sanitária de pescado.

Ocorre em águas não muito profundas, até cerca de 50 metros de profundidade. Encontrado tanto no Atlântico Oriental como no Ocidental, neste estendendo-se da Nova Escócia, no Canadá, à Argentina. Muito comum durante o verão no litoral sudeste do Brasil (Figueiredo e Menezes, 2000).
A Ordem Trypanorhyncha encontra-se composta por grande diversidade de espécies, todas parasitando peixes e invertebrados marinhos. Os parasitos adultos habitam o intestino de peixes elasmobrânquios (tubarões e raias) e as formas larvares são encontradas na cavidade celomática e na musculatura de peixes teleósteos, crustáceos e moluscos cefalópodes (Campbell e Beveridge, 1994).

Os cestóides da Ordem Trypanorhyncha, adquirem importância pelo aspecto desagradável que transferem ao consumidor, sendo os peixes descartados na indústria de beneficiamento ou nos serviços de inspeção, causando prejuízos econômicos. Pesquisas recentes informam que estes cestóides também podem ocasionar reações alérgicas em humanos (Rodero e Cuéllar, 1999; Vásquez-López et al., 2001).

* Faculdade de Medicina Veterinária, Universidade Federal Fluminense, Rua Vital Brazil Filho, 64, Vital Brazil, CEP 24230-340, Niterói, RJ, Brasil

** Laboratório de Helmintos Parasitos de Vertebrados, Instituto Oswaldo Cruz, FIOCRUZ, Avenida Brasil 4365, Manguinhos, CEP 21045-900, Rio de Janeiro, RJ, Brasil 
Com o objetivo de elucidar a biologia destes cestóides, que parasitam diversas espécies de peixes do litoral brasileiro, vários trabalhos foram publicados com enfoque taxonômico e higiênico-sanitário e, recentemente, estudos sobre a comunidade ecológica de metazoários parasitos de teleósteos capturados na costa brasileira detectaram a presença destes cestóides (São Clemente et al., 2004).

Registros anteriores de espécies da ordem Trypanorhyncha parasitando peixes do gênero Balistes sp. foram realizados, no Brasil, por São Clemente et al. (1995) em B. vetula e por Alves et al. (2005) em B. vetula e B. capriscus.

O gênero Callitetrarhynchus Pintner, 1931, tem sido registrado em uma ampla variedade de peixes e é constituído por duas espécies: C. gracilis Rudolphi, 1819 e C. speciosus Linton, 1897.

A espécie $C$. gracilis foi descrita pela primeira vez por Rudolphi, em 1819, sob a denominação de Anthocephalus gracilis. Pintner, em 1931 alterou a denominação do gênero para Callitetrarhynchus sp. No Brasil seu primeiro registro foi feito por Dollfus (1942) em Centropomus undecimalis, capturado na Ilha de Marajó, no estado do Pará (Pereira Jr., 1993).

Conforme Dollfus (1942), os cistos de Trypanorhyncha não são transmissíveis aos vertebrados homeotérmicos e o reencapsulamento das pós-larvas não ocorre em animais de sangue quente porém, segundo Deardoff et al. (1984) a localização das larvas de Trypanorhyncha na musculatura dos peixes pode produzir toxinas e consequentemente afetar o consumidor além do aspecto repugnante conferido ao peixe.

O presente trabalho objetiva o estudo dos metacestóides da Ordem Trypanorhyncha presente em Balistes capriscus Gmelin, 1789, comercializados nos municípios de Niterói e Rio de Janeiro, Brasil, apresentando os seus índices parasitários e sítios de infecção.

\section{Material e métodos}

De junho de 2005 a agosto de 2006, foram adquiridos 100 espécimes de peroá, Balistes capriscus Gmelin, 1789, em mercados de pescado dos municípios de Niterói e Rio de Janeiro. Os espécimes apresentaram variação de comprimento total entre 33,2 a $59,0 \mathrm{~cm}$ (44,5cm). De acordo com o comprimento total foram divididos em três classes de tamanho: $\mathrm{A}=30$ a $39,9 \mathrm{~cm} ; \mathrm{B}=40$ a $49,9 \mathrm{~cm}$ e $\mathrm{C}=50$ a $59,9 \mathrm{~cm}$.

Após a coleta os peixes foram transportados em recipientes isotérmicos contendo gelo para o Laboratório de Inspeção e Tecnologia de Pescado da Faculdade de Veterinária da Universidade Federal Fluminense, onde foram identificados, medidos, necropsiados e filetados.

Os plerocercos (larvas formadoras de blastocistos) dos cestóides de Trypanorhyncha foram coletados dos sítios de infecção, colocados em placa de Petri contendo água des-tilada, e liberados através do rompimento dos blastocistos, com o auxílio de estiletes, no estereomicroscópio. Em seguida foram contados e transferidos para o refrigerador (no mínimo 24 horas), possibilitando o relaxamento dos escólices e extroversão dos tentáculos.

Posteriormente, as larvas foram fixadas em AFA por, pelo menos, 24 horas, coradas pelo carmim de Langeron, diferenciadas em álcool clorídrico a $0,5 \%$, desidratadas pela série alcoólica crescente, clarificadas em creosoto de Faia e montadas entre lâmina e lamínula com bálsamo do Canadá ou mantidos em álcool 70 $\mathrm{GL}$, de acordo com Amato et al. (1991). A classificação dos cestóides da Ordem Trypanorhyncha foi realizada segundo Campbell e Beveridge (1994) e a determinação específica segundo Carvajal e Rego (1985).

As terminologias de ecologia parasitária seguem o proposto por Bush et al. (1997). Espécimes representativos dos parasitos foram depositados na Coleção Helmintológica do Instituto Oswaldo Cruz (CHIOC).

\section{Resultados}

Dos 100 peixes $B$. capriscus coletados $9 \%$ apresentaram duas espécies de plerocercos pertencentes a ordem Trypanorhyncha: Callitetrarhynchus gracilis e $C$. speciosus. Os índices parasitário de prevalência, intensidade média, amplitude média e abundância média de infecção, bem como os sítios de infecção e o número de depósito na CHIOC estão apresentados na Tabela 1. Os índices parasitários em relação aos intervalos de classe do comprimento total dos hospedeiros estão apresentados na Tabela 2.

Tabela 1: Prevalência (P), intensidade média (IM), abundância média (AM), amplitude de variação da intensidade de infecção $(\mathrm{Al})$, sítio de infecção e o número do depósito na $\mathrm{CHIOC}$ dos plerocercos da ordem Trypanorhyncha coletados de Balistes capriscus, comercializados no estado do Rio de Janeiro, entre junho de 2005 a agosto de 2006

\begin{tabular}{ccccccc}
\hline $\begin{array}{c}\text { Espécies de plerocercos de } \\
\text { Trypanorhyncha }\end{array}$ & $\mathrm{P}(\%)$ & $\mathrm{IM}$ & $\mathrm{AM}$ & $\mathrm{Al}$ & $\begin{array}{c}\text { Sítio de } \\
\text { infecção }\end{array}$ & $\begin{array}{c}\mathrm{N} N \\
\mathrm{CHIOC}\end{array}$ \\
\hline Callitetrarhynchus gracilis & 9 & 14,4 & 0,13 & $1-$ & $\begin{array}{c}\text { fígado } \\
\text { mesentério }\end{array}$ & 35640 \\
Callitetrarhynchus speciosus & 2 & 1 & 0,02 & - & mesentério & 35641 \\
\hline
\end{tabular}

Tabela 2: Relação entre as classes de tamanho do peixe teleósteo Balistes capriscus, comercializados no estado do Rio de Janeiro, entre junho de 2005 a agosto de 2006, e as espécies de cestóides da ordem Trypanorhyncha, indicando a prevalência $(P)$, intensidade média (IM) e abundância média de infecção (AM)

\begin{tabular}{ccccccc}
\hline & \multicolumn{3}{c}{$\begin{array}{c}\text { Espécies de cestóides } \\
\text { Callitetrarhynchus } \\
\text { gracilis }\end{array}$} & \multicolumn{3}{c}{$\begin{array}{c}\text { Calletrarhynchus } \\
\text { speciosus }\end{array}$} \\
\hline Classes de tamanho de & $\mathrm{P}(\%)$ & $\mathrm{IM}$ & $\mathrm{AM}$ & $\mathrm{P}(\%)$ & $\mathrm{IM}$ & $\mathrm{AM}$ \\
Balistes capriscus & 1 & 1 & 1 & - & - & 0 \\
A & - & - & 0 & - & - & 0 \\
B & 8 & 16 & 0,6 & 2 & 1 & 0,16 \\
C & & & & & & \\
\hline
\end{tabular}

A: $30-39,9 \mathrm{~cm}$ de comprimento total; B: $40-49,9 \mathrm{~cm}$; C: $50-59,9 \mathrm{~cm}$. 
Observamos que os índices parasitários apresentados por C. gracilis na classe de menor tamanho foram menores, com exceção da abundância média que foi maior nesta classe. Em relação a $C$. speciosus este somente ocorreu na classe de maior tamanho. Vale observar que na classe intermediária houve ausência de parasitos.

Este é o primeiro registro das espécies Callitetrarhynchus gracilis e C. speciosus em Balistes capriscus.

\section{Discussão e conclusão}

Comparando os registros de Alves et al. (2005) com os resultados deste trabalho, os autores relataram larvas de Callitetrarhynchus sp. parasitando $B$. capricus com $P=16,7 \%$, $\mathrm{IM}=6,6$ e $\mathrm{AM}=1,1$, sendo superior à encontrada na presente pesquisa. Os autores op. cit. não determinaram os parasitos ao nível de espécie, porém informaram o número de depósito dos espécimes na CHIOC (36479 e 36480) e após consulta dos mesmos foi observado que não apresentavam os tentáculos extrovertidos, impossibilitando a determinação específica.

Em outra espécie pertencente ao gênero Balistes sp., São Clemente et al. (1995) observaram que em 50 amostras de B. vetula, $88 \%$ encontravam-se parasitadas por cestóides das espécies $C$. speciosus e $C$. gracilis. Os autores não citaram os índices parasitários para cada espécie, ressaltando que o parasitismo por este grupo de cestóides tem caráter acumulativo, uma vez que os peixes de maior tamanho encontravam-se mais parasitados.

\section{Referências}

ALVES, D. R.; PARAGUASSU, A.R.; LUQUE, J.L. Community ecology of the metazoan parasite of the grey triggerfish, Balites capriscus Gmelin, 1789 and queen triggerfish B. vetula Linnaeus, 1758 (Osteichthyes: Balistidae) from the state of Rio de Janeiro, Brazil. Revista Brasileira de Parasitologia Veterinária, v.14, n. 2, p. 71-77, 2005.

AMATO, J.F.R.; BOEGER, W.A.; AMATO, S.B. Protocolos para o Laboratório em Coleta e Processamento de Parasitos de Pescado. Seropédica: Imprensa Universitária, UFRRJ, Rio de Janeiro, 1991.

BUSH, A.O.; LAFFERTY, K.D.; LOTZ, J.M.; SHOSTAK, A.W. Parasitology meets ecology on its own terms. Magnolis et al., Reviseted. Journal of Parasitology, v. 83, p. 575-583, 1997.

CAMPBELL, R.A.; BEVERIDGE, I. Order Trypanorhyncha Diesing, 1863. In: Chapter 7. Keys to the Cestodes Parasites of Vertebrates Eds. L.F. Khalil, Jones, A. \& Bray, R.A. Wallingford, UK : Commonwealth Agriculture Bureaux International, 1994. p. 51-148.

CARVAJAL, J.; REGO, A.A. Critical studies on the genus Callitetrarhynchus (Cestoda: Trypanorhyncha) with recognition of Rhychobothrium speciosum Linton, 1897 as a valid species of the genus Callitetharhynchus. Systemat. Parasitol., v. 7, p. 161-162,1985.

DEARDOFF, T.L.; RAYBOURNE, R.B.; MATTIS, T.E. Infections with Trypanorhyncha plerocerci (Cestoda) in hawaiian fishes of commercial importance. Sea Grant Quartely, v. 6, n. 3, p. 1-6, 1984.

DOLLFUSS, R. PH. Etudes critiques sur les Tétrarhynches du Museúm de Paris. Arquives du Museúm National d'Histoire Naturelle, v. 19, p. 1-466, 1942.
$\mathrm{Na}$ presente pesquisa a prevalência maior foi verificada na classe de maior tamanho, para as duas espécies de larvas encontradas, no entanto deve-se evitar generalizações relacionadas com a influência do tamanho do peixe sobre a composição qualitativa e quantitativa das infracomunidades parasitárias, como postulado por Saad-Fares e Combes (1992) até porque no intervalo de classe intermediário não houve presença dos referidos parasitos e a abundância maior para C. gracilis ocorreu na classe de menor tamanho.

O número de metacestóides coletados indica que, por serem peixes predadores, ocupam nível intermediário no ciclo das ictioparasitoses, como parte da dieta de hospedeiros definitivos.

Cabe ressaltar a importância da conscientização pelos profissionais de Vigilância e Inspeção Sanitária e dos demais relacionados com a cadeia de produção, desde a captura até o consumo, com respeito as ictioparasitoses e formas de profilaxia, implementando programas de educação sanitária em todos os níveis.

Sugere-se a aplicação do plano de Análise de Perigos e Pontos Críticos de Controle (APPCC) em todos os pontos da cadeia de produção com a finalidade de eliminar, prevenir ou reduzir os riscos, garantindo produto com qualidade e segurança, neste caso com a evisceração a bordo ou nas indústrias, com o devido destino do descarte, assim como o desenvolvimento de pesquisas com cestóides da ordem Trypanorhyncha para avaliação de seu potencial zoonótico em relação às suas toxinas, que algumas vezes têm sido citadas causando alergia em humanos.

FIGUEIREDO, J.L.; MENEZES, N.A. Manual de peixes marinhos do Sudeste do Brasil, vol. VI. Teleostei (5). São Paulo: Museu de Zoologia, Universidade de São Paulo, 2000.

PEREIRA JR., J. O complexo de espécies de Trypanorhyncha (Cestoda), em corvinas Micropogonias furnieri do litoral do Rio Grande do Sul. Arquivos da Faculdade de Veterinária da UFRGS, v. 21, p. 58-70, 1993.

RODERO, M.; CUÉLLAR, C. Humoral responses induced by Gymnorhynchus gigas extracts in BALB/ C mice. Journal of Helminthology, v. 73, p. 239-273, 1999.

SAADE-FARES, A.; COMBES, C. Abundance/host size relationships in a fish trematode community. Journal of Helminthology, v. 66, p. 187-192, 1992.

SÃO CLEMENTE, S.C.; KNOFF, M.; PADOVANI, R.E.S.; LIMA, F.C.; GOMES, D.C. Cestóides parasitos de congro-rosa, Genypterus brasiliensis Regan, 1903 comercializados nos municípios de Niterói e Rio de Janeiro, Brasil. Rev. Bras. Parasitologia Vet., v. 13, n. 3, p. 97-102, 2004.

SÃO CLEMENTE, S.C.; LIMA, F.C.; UCHOA, C.M.A . Parasitos de B. vetula (L.) e sua importância na inspeção do pescado. Revista Brasil. Ciên. Vet., v. 2, n. 2, p. 39-41, 1995.

VÁZQUEZ-LÓPEZ, C.; ARMAS-SERRA, C.; RODRÍGUES-CAABEIRO, F. Gymnorhynchus gigas: taxonomía, morfología, biología y aspectos sanitarios. Analecta Veterinaria, v. 21, n. 2, p. 38-49, 2001. 